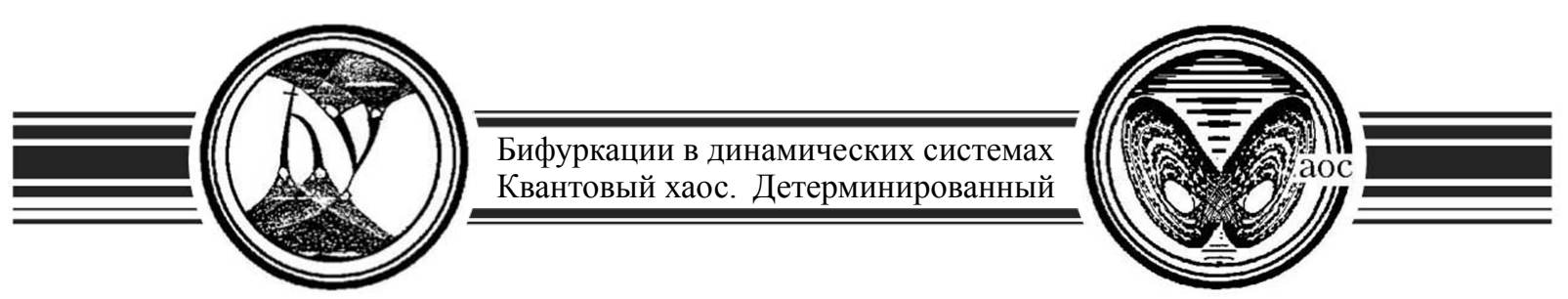

УДК 517.9

https://doi.org/10.18500/0869-6632-2020-28-3-274-281

\title{
Метод выделения характерных фаз поведения в системах со сложной топологией аттрактора, находящихся вблизи границы обобщенной синхронизации
}

\section{О. И. Москаленко, А. А. Короновский, В. А. Ханадеев}

Саратовский национальный исследовательский государственный университет имени Н.Г. Чернышевского, Региональный научно-образовательный математический центр «Математика технологий будущего» Россия, 410012 Саратов, Астраханская, 83

E-mail: o.i.moskalenko@gmail.com, alexey.koronovskii@gmail.com, v.a.hanadeev@gmail.com Автор для переписки Ольга Игоревна Москаленко, o.i.moskalenko@gmail.com Поступила в редакцию 19.03.2020, принята к публикации 07.05.2020, опубликована 30.06.2020

Целью работы является разработка универсального метода выделения характерных фаз поведения систем со сложной топологией аттрактора, находящихся в режиме перемежающейся обобщенной синхронизации. Метод основан на анализе расположения изображающих точек на аттракторах взаимодействующих систем, связанных однонаправленно или взаимно. Результатом работы является проверка работоспособности предложенного метода на системах с однонаправленной связью (двух однонаправленно связанных осцилляторах Лоренца, находящихся в хаотическом режиме), допускающих анализ перемежаемости при помощи метода вспомогательной системы. В работе установлено, что перескок изображающих точек на разные листы аттракторов взаимодействующих систем предшествует возникновению турбулентной фазы поведения, диагностируемой при помощи метода вспомогательной системы. При помощи обоих методов произведен расчет статистических характеристик перемежаемости (распределений длительностей ламинарных фаз при нескольких фиксированных значениях параметра связи) и проведено их сопоставление друг с другом. Установлено, что во всех рассмотренных случаях результаты обоих методов практически в точности совпадают, при этом распределения длительностей ламинарных фаз подчиняются экспоненциальным законам, что не характерно для систем с достаточно простой топологией аттрактора. Сделано предположение о том, что в системах со сложной топологией аттрактора наблюдается новый тип перемежаемости - перемежаемость перескоков.

Ключевые слова: сложная топология аттрактора, обобщенная синхронизация, метод вспомогательной системы, метод перескоков, перемежаемость.

Образец цитирования: Москаленко О.И., Короновский А.А., Ханадеев В.А. Метод выделения характерных фаз поведения в системах со сложной топологией аттрактора, находящихся вблизи границы обобщенной синхронизации // Известия вузов. ПНД. 2020. Т. 28, № 3. С. 274-281. https://doi.org/10.18500/0869-6632-2020-28-3-274-281

Статья опубликована на условиях лицензии Creative Commons Attribution License (CC-BY 4.0).

Финансовая поддержка. Работа выполнена при финансовой поддержке Совета по грантам Президента Российской Федерации для государственной поддержки молодых российских ученых - докторов наук (проект № МД-21.2020.2). 


\title{
Method for characteristic phase detection in systems with complex topology of attractor being near the boundary of generalized synchronization
}

\author{
O. I. Moskalenko, A. A. Koronovskii, V. A. Khanadeev \\ Saratov State University \\ Regional Scientific and Educational Mathematical Center \\ «Mathematics of Future Technologies» \\ 83, Astrakhanskaya str., Saratov 410012, Russsia \\ E-mail: o.i.moskalenko@gmail.com, alexey.koronovskii@gmail.com,v.a.hanadeev@gmail.com \\ Correspondence should be addressed to Olga I. Moskalenko, o.i.moskalenko@gmail.com \\ Received 19.03.2020, accepted 07.05.2020, published 30.06.2020
}

\begin{abstract}
The aim of the paper consists in the development of universal method for the detection of characteristic phases of the behavior in systems with complex topology of attractor being in the regime of intermittent generalized synchronization. The method is based on an analysis of the location of representation points on the attractors of interacting systems coupled unidirectionally or mutually. The result of this work is the verification of the performance of the proposed method on systems with unidirectional coupling (two unidirectionally coupled Lorenz oscillators being in chaotic regime) that allow the analysis of intermittency using the auxiliary system method. It was found that the jump of the representation points to different sheets of attractors of interacting systems precedes the appearance of the turbulent phase of the behavior detected using the auxiliary system method. Using both methods, the statistical characteristics of intermittency, i.e. the distributions of the laminar phase lengths for several fixed values of the coupling parameter, were calculated and they were compared with each other. It was found that in all considered cases the results of both methods almost exactly coincide with each other, while the distributions of the laminar phase lengths obey the exponential laws, which is not typical for systems with a simple enough topology of attractor. It was assumed that in systems with a complex topology of attractor a new type of intermittency called by jump intermittency is observed.
\end{abstract}

Key words: complex topology of attractor, generalized synchronization, auxiliary systems method, jump method, intermittency.

Reference: Moskalenko O.I., Koronovskii A.A., Khanadeev V.A. Method for characteristic phase detection in systems with complex topology of attractor being near the boundary of generalized synchronization. Izvestiya VUZ. Applied Nonlinear Dynamics, 2020, vol. 28, no. 3, pp. 274-281. https://doi.org/10.18500/0869-6632-2020-28-3-274-281

This is an open access article distributed under the terms of Creative Commons Attribution License (CC-BY 4.0).

Acknowledgements. This work was financially supported by the Grant Council of the President of the Russian Federation for the state support of young Russian scientists - doctors of sciences (project No. MD-21.2020.2).

Обобщенная синхронизация представляет собой один из наиболее интересных и наименее изученных типов хаотической синхронизации [1-3]. Данный тип синхронизации означает наличие функциональной связи (функционала) между состояниями взаимодействующих систем (в том числе, узловых элементов сетей), связанных однонаправленно или взаимно, и может быть диагностирован при помощи метода ближайших соседей [1], метода фазовых трубок [2], метода расчета спектра показателей Ляпунова [4], а для однонаправленно связанных (ведущей и ведомой) систем и древовидных сетей - еще и при помощи метода вспомогательной системы [5].

На границе обобщенной синхронизации во всех случаях наблюдается перемежающееся поведение, определить характеристики которого в случае однонаправленной связи можно также при помощи метода вспомогательной системы [6]. Для этого необходимо ввести в рассмотрение вспомогательную систему, идентичную ведомой системе по управляющим параметрам, и проанализировать разность между состояниями этих систем с течением времени. Понятно, что в режиме перемежаемости эта разность представляет собой чередование участков с синхронной (ламинарные фазы) и асинхронной (турбулентные фазы) динамикой. Определить тип перемежаемости возможно, проанализировав статистику длительностей ламинарных фаз - тех временных 
интервалов, где абсолютная величина этой разности оказывается меньше некоторой наперед заданной величины $\Delta$. Для систем с взаимной связью метод вспомогательной системы оказывается неработоспособным [7], что требует разработки других методов анализа перемежаемости в таких системах.

Особым классом динамических систем являются системы с так называемой сложной (двулистной) топологией аттрактора, как например, у системы Лоренца, генераторов Чуа с аттрактором типа двойной спирали и т.д. [8]. Можно ожидать, что переход к режиму обобщенной синхронизации в таких системах имеет особенности, отличные от последних в системах с достаточно простой топологией аттрактора, что сделает возможным диагностику этого режима при помощи методов и подходов, неприменимых в случае более простых систем.

В настоящей работе мы предлагаем метод выделения характерных фаз поведения, основанный на анализе расположения изображающих точек на аттракторах взаимодействующих систем со сложной топологией аттрактора. Как показано ниже, перескок изображающих точек на разные листы аттракторов этих систем предшествует возникновению турбулентной фазы, диагностируемой при помощи метода вспомогательной системы.

В качестве объекта исследования в работе была выбрана система двух однонаправленно связанных осцилляторов Лоренца [9]:

$$
\begin{aligned}
& \dot{x}_{1}=\sigma\left(y_{1}-x_{1}\right), \\
& \dot{y}_{1}=r_{1} x_{1}-y_{1}-x_{1} z_{1}, \\
& \dot{z}_{1}=-b z_{1}+x_{1} y_{1}, \\
& \dot{x}_{2}=\sigma\left(y_{2}-x_{2}\right)+\varepsilon\left(x_{1}-x_{2}\right), \\
& \dot{y}_{2}=r_{2} x_{2}-y_{2}-x_{2} z_{2}, \\
& \dot{z}_{2}=-b z_{2}+x_{2} y_{2},
\end{aligned}
$$

где $\mathbf{x}_{1,2}=\left(x_{1,2}, y_{1,2}, z_{1,2}\right)$ - векторы состояний взаимодействующих систем; $\sigma=10, b=2$, $r_{1}=40$ и $r_{2}=35$ - управляющие параметры; $\varepsilon$ - параметр связи. Как показали проведенные расчеты, режим обобщенной синхронизации в данном случае возникает при $\varepsilon=9.95$.

Ниже границы обобщенной синхронизации, как отмечалось выше, наблюдается перемежающееся поведение. Для определения статистических характеристик перемежаемости были использованы как метод вспомогательной системы, так и предлагаемый подход, основанный на анализе расположения изображающих точек на аттракторе. Так как структура аттракторов взаимодействующих систем является двулистной, можно предположить, что возникновение турбулентной фазы вблизи границы обобщенной синхронизации может быть связано с перескоком изображающих точек с одного листа хаотического аттрактора на другой: если состояния обеих (ведущей и ведомой) взаимодействующих систем находятся на одном и том же листе аттрактора, в системе реализуется фаза синхронного поведения, в противном случае возникает асинхронная (турбулентная) фаза. Вышесказанное иллюстрирует рис. 1, где представлены $(a)$ фрагменты временных реализаций ведущей и ведомой систем Лоренца, содержащие ламинарную и турбулентную фазы поведения, $(b)$ разность между состояниями ведомой и вспомогательной систем, соответствующая этим фрагментам, $(c)$ и $(d)$ аттракторы ведомой и вспомогательной систем с отмеченными на них фазовыми траекториями, соответствующими этим фрагментам. Из рисунка видно, что перескок изображающих точек связанных осцилляторов с одинаковых листов аттракторов на разные предшествует возникновению турбулентной фазы поведения. Аналогичное поведение наблюдается и для других интервалов времени, соответствующих характерным фазам поведения системы. На основе анализа временной реализации длительностью $L=3$ млн единиц безразмерного времени (порядка 10000 ламинарных фаз) статистически установлено, 

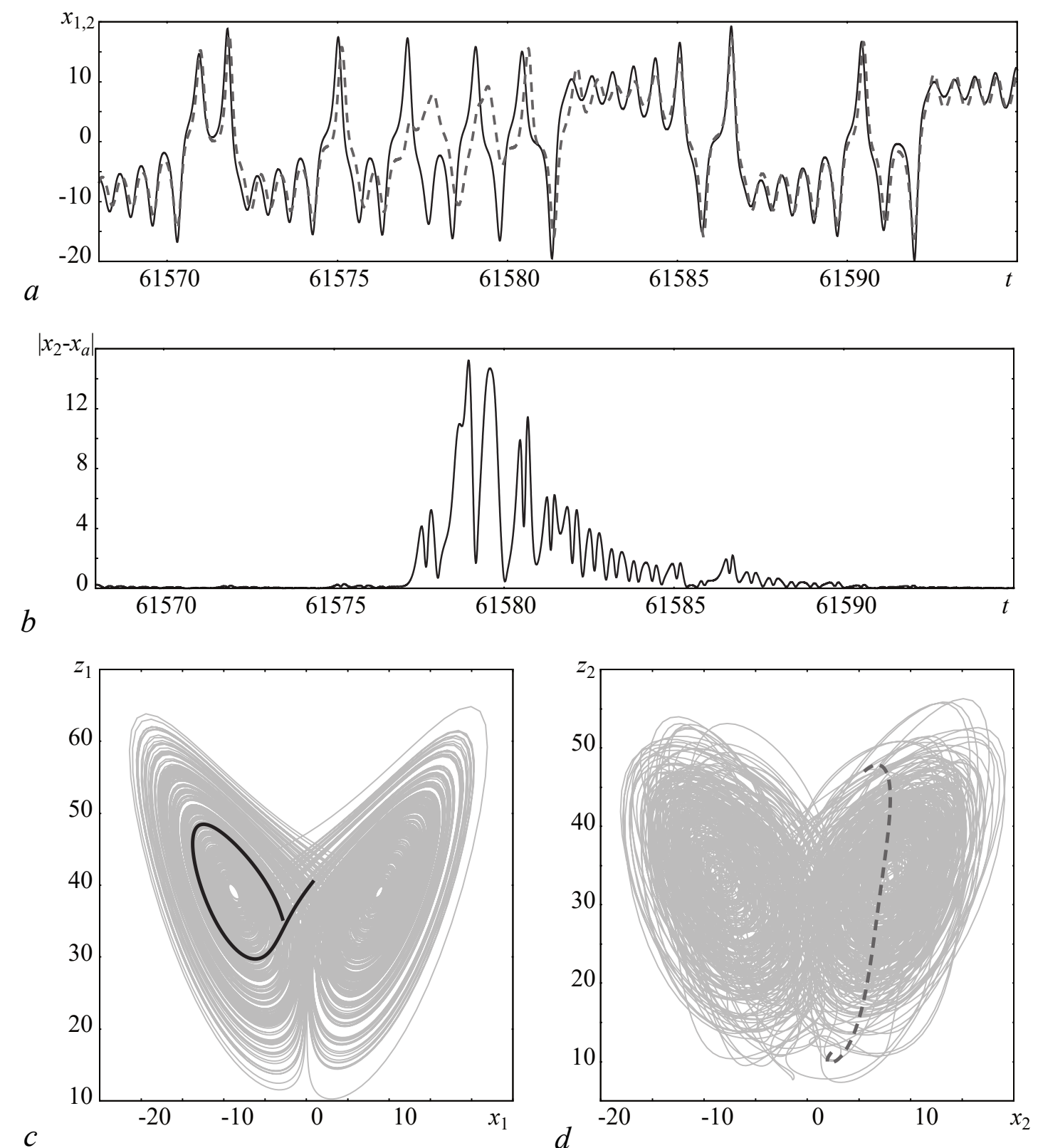

Рис. 1. $a$-Фрагменты временных реализаций ведущей $x_{1}(t)$ (сплошная линия) и ведомой $x_{2}(t)$ (пунктирная линия) однонаправленно связанных систем Лоренца (1). $b$ - Временная динамика абсолютной величины разности между $x$-координатами ведомой и вспомогательной систем. Хаотические аттракторы ведущей $(c)$ и ведомой $(d)$ систем Лоренца (светло-серый) и фазовые траектории, соответствующие началу турбулентной фазы в ведущей и ведомой системах. Значение параметра связи $\varepsilon=9.6$

Fig. 1. $a$-Fragments of time realizations of the drive $x_{1}(t)$ (solid line) and response $x_{2}(t)$ (dashed line) unidirectionally coupled Lorentz systems (1). $b$ - Time dynamics of the absolute value of the difference between $x$-coordinates of the response and auxiliary systems. Chaotic attractors of the drive $(c)$ and response $(d)$ Lorentz systems (light grey) and phase trajectories corresponding to the beginning of the turbulent phase in the drive and response systems. The coupling parameter value is $\varepsilon=9.6$

Москаленко О.И., Короновский А.А., Ханадеев В.А.

Известия вузов. ПНД, 2020, т. 28, № 3 


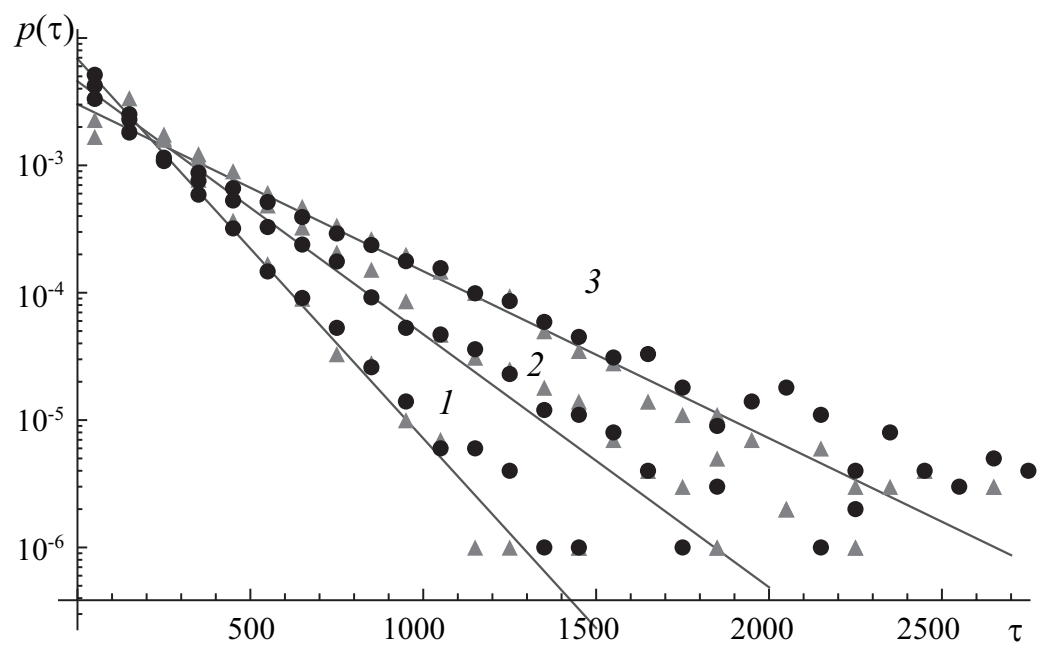

Рис. 2. Нормированные распределения длительностей ламинарных фаз двух однонаправленно связанных систем Лоренца (1), рассчитанные при помощи метода вспомогательной системы (кружки) и анализа расположения точек на аттракторе (треугольники), и их теоретические аппроксимации экспоненциальными законами (2), полученные при фиксированных значениях параметра связи. Параметры аппроксимации выбраны следующими: $1-\varepsilon=9.3, T=145.7$; $2-\varepsilon=9.6, T=218.6 ; 3-\varepsilon=9.9, T=331.2$

Fig. 2. Normalized distributions of the laminar phase lengths of two unidirectionally coupled Lorentz systems (1) calculated by means of auxiliary system approach (circles) and analysis of location of points on attractor (triangles) and their theoretical approximations by the exponential laws (2) obtained for fixed values of the coupling parameter. Parameters of approximation have been selected as follows: $1-\varepsilon=9.3, T=145.7 ; 2-\varepsilon=9.6, T=218.6 ; 3-\varepsilon=9.9, T=331.2$

что в 99.93\% случаев перескоки изображающих точек взаимодействующих систем с одинаковых листов хаотических аттракторов на разные предшествуют возникновению турбулентных фаз, что говорит о возможности применения данного подхода для анализа статистических характеристик перемежаемости вблизи границы обобщенной синхронизации.

На рис. 2 представлены статистические характеристики длительностей ламинарных фаз, рассчитанные обоими способами: распределения длительностей ламинарных фаз при нескольких значениях параметра связи. Данные численного моделирования показаны символами (кружками - для метода вспомогательной системы, треугольниками - для метода, основанного на анализе расположения изображающих точек на аттракторе ${ }^{1}$ ), теоретические аппроксимации сплошными линиями. Из рисунка видно, что во всех рассмотренных случаях статистические характеристики, полученные двумя различными способами, практически в точности совпадают друг с другом. Кроме того, нетрудно заметить, что они подчиняются экспоненциальным законам

$$
p(\tau)=\frac{1}{T} \exp \left(-\frac{\tau}{T}\right)
$$

( $T$ - средняя длительность ламинарных фаз при фиксированных значениях управляющих параметров), имеющим место в режиме индуцированной шумом перемежаемости в бистабильных системах [10], что не характерно для перемежаемости вблизи границы обобщенной синхронизации в системах с достаточно простой топологией аттрактора. Можно предположить, что в системах со сложной топологией аттрактора на границе обобщенной синхронизации имеет место новый тип перемежающегося поведения (перемежаемость перескоков), статистические характеристики которого подчиняются экспоненциальным законам.

\footnotetext{
${ }^{1}$ Под ламинарной фазой, диагностируемой при помощи метода, основанного на анализе расположения изображающих точек на аттракторе, понимается интервал времени между двумя последовательными перескоками изображающих точек с одинаковых листов хаотических аттракторов взаимодействующих осцилляторов на разные.
} 
Таблица. Среднеквадратичные отклонения распределений длительностей ламинарных фаз, полученных численно при помощи метода вспомогательной системы (метод 1) и анализа расположения точек на аттракторе (метод 2), от теоретической закономерности (2) при значениях $T$, указанных в подрисуночной подписи к рис. 2

Table. Standard deviations of the distributions of the laminar phase lengths obtained numerically by the auxiliary system method (method 1 ) and the analysis of the location of representation points on the attractor (method 2) from the theoretical regularity (2) for the values of $T$ indicated in the figure caption to Fig. 2

\begin{tabular}{|l|l|l|}
\hline & Метод 1 & Метод 2 \\
\hline$\varepsilon=9.3$ & 0.00008 & 0.00038 \\
\hline$\varepsilon=9.6$ & 0.00013 & 0.00030 \\
\hline$\varepsilon=9.9$ & 0.00014 & 0.00018 \\
\hline
\end{tabular}

Среднеквадратичные отклонения данных численного моделирования от теоретических закономерностей (2) представлены в Таблице.

Из данных таблицы видно, что, хотя предложенный метод по мере среднеквадратичного отклонения от экспоненциальной аппроксимации несколько уступает классическому методу вспомогательной системы, степень отклонения данных численного моделирования от теоретической закономерности остается малой. Более того, с ростом параметра связи отклонение метода вспомогательной системы увеличивается, а метода, основанного на анализе расположения точек на аттракторе, наоборот, уменьшается, и при $\varepsilon=9.9$ оба метода оказываются практически равнозначными. Следует также отметить, что предложенный подход может найти применение при анализе перемежаемости даже в том случае, когда реализация метода вспомогательной системы не представляется возможной, например, в случае взаимной связи между системами $[11,12]$, что является дополнительным свидетельством эффективности предложенного метода.

Таким образом, в настоящей работе предложен метод выделения ламинарных и турбулентных фаз взаимодействующих систем со сложной топологией аттрактора, находящихся в режиме перемежающейся обобщенной синхронизации. Эффективность метода проверена путем сопоставления его результатов с результатами метода вспомогательной системы, получено хорошее соответствие между ними.

\section{Библиографический список}

1. Rulkov N.F., Sushchik M.M., Tsimring L.S. and Abarbanel H.D.I. Generalized synchronization of chaos in directionally coupled chaotic systems // Phys. Rev. E. 1995. Vol. 51. P. 980-994. doi:10.1103/PhysRevE.51.980.

2. Koronovskii A.A., Moskalenko O.I., Hramov A.E. Nearest neighbors, phase tubes, and generalized synchronization // Phys. Rev. E. 2011. Vol. 84, no. 3. 037201. doi:10.1103/PhysRevE.84.037201.

3. Moskalenko O.I., Koronovskii A.A., Hramov A.E., Boccaletti S. Generalized synchronization in mutually coupled oscillators and complex networks // Phys. Rev. E. 2012. Vol. 86. 036216. doi:10.1103/PhysRevE.86.036216.

4. Pyragas K. Conditional Lyapunov exponents from time series // Phys. Rev. E. 1997. Vol. 56, no. 5. P. 5183-5188. doi:10.1103/PhysRevE.56.5183.

5. Abarbanel H.D.I., Rulkov N.F., Sushchik M. Generalized synchronization of chaos: The auxiliary system approach // Phys. Rev. E. 1996. Vol. 53, no. 5. P. 4528-4535.

doi:10.1103/PhysRevE.53.4528. 
6. Hramov A.E., Koronovskii A.A. Intermittent generalized synchronization in unidirectionally coupled chaotic oscillators // Europhysics Letters. 2005. Vol. 70, no. 2. P. 169-175. doi:10.1209/ep1/i2004-10488-6.

7. Moskalenko O.I., Koronovskii A.A., Hramov A.E. Inapplicability of an auxiliary-system approach to chaotic oscillators with mutual-type coupling and complex networks // Phys. Rev. E. 2013. Vol. 87, no. 6. 064901. doi:10.1103/PhysRevE.87.064901.

8. Кузнеиов С.П. Динамический хаос. М.: Физматлит, 2006. 356 с.

9. Zheng Z., Wang X., Cross M.C. Transitions from partial to complete generalized synchronizations in bidirectionally coupled chaotic oscillators // Phys. Rev. E. 2002. Vol. 65, no. 5. 056211. doi:10.1103/PhysRevE.65.056211.

10. Moskalenko O.I., Koronovskii A.A., Zhuravlev M.O., Hramov A.E. Characteristics of noiseinduced intermittency // Chaos, Solitons \& Fractals. 2018. Vol. 117. P. 269-275. doi:10.1016/j.chaos.2018.11.001.

11. Москаленко О.И., Ханадеев В.А., Короновский А.А. Метод диагностики обобщенной синхронизации в системах со сложной топологией хаотического аттрактора // Письма в ЖТФ. 2018. T. 44, № 19. С. 87-95. doi:10.21883/PJTF.2018.19.46687.17391.

12. Москаленко О.И., Короновский А.А., Ханадеев В.А. Перемежающееся поведение на границе обобщенной синхронизации во взаимно связанных системах со сложной топологией аттрактора // ЖТФ. 2019. Т. 89, № 3. С. 338-341. doi:10.21883/JTF.2019.03.47165.325-18.

\section{References}

1. Rulkov N.F., Sushchik M.M., Tsimring L.S. and Abarbanel H.D.I. Generalized synchronization of chaos in directionally coupled chaotic systems. Phys. Rev. E, 1995, vol. 51, pp. 980-994. doi:10.1103/PhysRevE.51.980.

2. Koronovskii A.A., Moskalenko O.I., Hramov A.E. Nearest neighbors, phase tubes, and generalized synchronization. Phys. Rev. E, 2011, vol. 84, no. 3, 037201. doi:10.1103/PhysRevE.84.037201.

3. Moskalenko O.I., Koronovskii A.A., Hramov A.E., Boccaletti S. Generalized synchronization in mutually coupled oscillators and complex networks. Phys. Rev. E, 2012, vol. 86, 036216. doi:10.1103/PhysRevE.86.036216.

4. Pyragas K. Conditional Lyapunov exponents from time series. Phys. Rev. E, 1997, vol. 56, no. 5 , pp. 5183-5188. doi:10.1103/PhysRevE.56.5183.

5. Abarbanel H.D.I., Rulkov N.F., Sushchik M. Generalized synchronization of chaos: The auxiliary system approach. Phys. Rev. E, 1996, vol. 53, no. 5, pp. 4528-4535. doi:10.1103/PhysRevE.53.4528.

6. Hramov A.E., Koronovskii A.A. Intermittent generalized synchronization in unidirectionally coupled chaotic oscillators. Europhysics Letters, 2005, vol. 70, no. 2, pp. 169-175. doi:10.1209/epl/i2004-10488-6.

7. Moskalenko O.I., Koronovskii A.A., Hramov A.E. Inapplicability of an auxiliary-system approach to chaotic oscillators with mutual-type coupling and complex networks. Phys. Rev. E, 2013, vol. 87, no. 6, 064901. doi:10.1103/PhysRevE.87.064901.

8. Kuznetsov S.P. Dynamical Chaos. M.: Fizmatlit, 2006, 356 p.

9. Zheng Z., Wang X., Cross M.C. Transitions from partial to complete generalized synchronizations in bidirectionally coupled chaotic oscillators. Phys. Rev. E, 2002, vol. 65, no. 5, 056211. doi:10.1103/PhysRevE.65.056211. 
10. Moskalenko O.I., Koronovskii A.A., Zhuravlev M.O., Hramov A.E. Characteristics of noiseinduced intermittency. Chaos, Solitons \& Fractals, 2018, vol. 117, pp. 269-275.

doi:10.1016/j.chaos.2018.11.001.

11. Moskalenko O.I., Khanadeev V.A., Koronovskii A.A. A diagnostic technique for generalized synchronization in systems with a complex chaotic attractor topology. Tech. Phys. Lett., 2018, vol. 44, no. 10, pp. 894-897. doi:10.1134/S1063785018100115.

12. Moskalenko O.I., Koronovskii A.A., Khanadeev V.A. Intermittency at the boundary of generalized synchronization in mutually coupled systems with complex attractor topology. Technical Physics, 2019, vol. 64, no. 3, pp. 302-305. doi:10.1134/S1063784219030198.

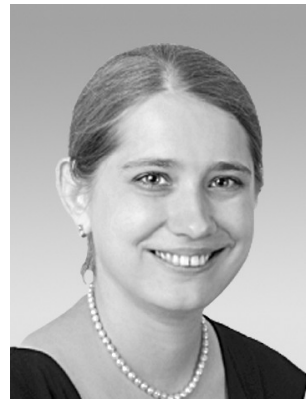

Москаленко Ольга Игоревна - родилась в Саратове (1984), окончила факультет нелинейных процессов СГУ (2006), кандидат физико-математических наук (2008), доцент (2012), доктор физико-математических наук (2017). Профессор кафедры физики открытых систем СГУ, начальник управления научной деятельности СГУ. Область научных интересов - исследование классической и хаотической синхронизации и явлений, имеющих место на ее границе, в нелинейных системах; применение непрерывного вейвлет- и фурье-анализа к проблеме хаотической синхронизации в системах с малым числом степеней свободы и пространственно-распределенных средах; изучение обобщенной синхронизации в системах с однонаправленной и взаимной связью и ее возможных приложений; влияние шума на установление синхронных режимов и характеристики перемежаемости; применение хаотической синхронизации для скрытой передачи информации; анализ хаотической синхронизации в сложных сетях; разработка методов анализа поведения систем по временным рядам и их применение к живым системам. Автор около 150 статей в центральных реферируемых отечественных и зарубежных журналах.

Россия, 410012 Саратов, Астраханская, 83

Саратовский государственный университет имени Н.Г. Чернышевского

E-mail: o.i.moskalenko@gmail.com

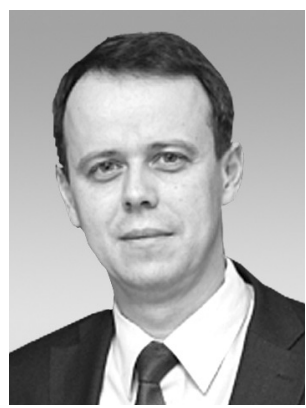

Короновский Алексей Александрович - родился в Саратове (1972). Окончил физический факультет Саратовского государственного университета (1995), доктор физикоматематических наук (2007), заведующий кафедрой физики открытых систем ФГБОУ ВО «Саратовский национальный исследовательский государственный университет имени Н.Г. Чернышевского». Область научных интересов - динамический хаос, нелинейная динамика и ее проявления в различных сферах человеческой деятельности. Автор научных монографий и статей в отечественных и зарубежных рецензируемых журналах.

Россия, 410012 Саратов, Астраханская, 83

Саратовский государственный университет имени Н.Г. Чернышевского

E-mail: alexey.koronovskii@gmail.com

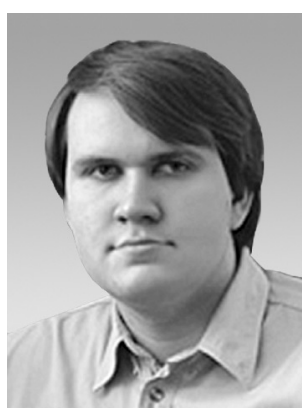

Ханадеев Владислав Андреевич - родился в Саратове (1995). Окончил бакалавриат (2017) и магистратуру (2019) факультета нелинейных процессов СГУ, в настоящее время является аспирантом кафедры физики открытых систем первого года обучения. Область научных интересов - динамический хаос, хаотическая синхронизация, системы со сложной топологией аттрактора. Автор двух статей в отечественных и зарубежных рецензируемых научных журналах, участник всероссийских и международных научных конференций.

Россия, 410012 Саратов, Астраханская, 83

Саратовский государственный университет имени Н.Г. Чернышевского

E-mail: v.a.hanadeev@gmail.com 